\title{
Germ-line sequence variants of PTEN do not have an important role in hereditary and non-hereditary prostate cancer susceptibility
}

\author{
Chunmei C Xie ${ }^{1,2}$, Lingyi Lu ${ }^{1,3}$, Jielin Sun ${ }^{1,4}$, S Lilly Zheng ${ }^{1,4}$, William B Isaacs ${ }^{5}$, Henrik Gronberg ${ }^{6}$ and \\ Jianfeng $\mathrm{Xu}^{1,4}$
}

PTEN (phosphatase and tensin homolog deleted on chromosome 10) functions as a major tumor suppressor gene and is frequently deleted in different types of tumors including prostate cancer (PCa). It was hypothesized that germ-line genetic changes of PTEN affect susceptibility to PCa. Both common (with a minor allele frequency $\geqslant 5 \%$ ) and rare (with a minor allele frequency $<5 \%$ ) germ-line variants of PTEN were comprehensively evaluated. A total of 15 germ-line variants were identified by re-sequencing the PTEN gene, including $5^{\prime}$ untranslated region, all nine exons, exon-intron junctions and $3^{\prime}$ untranslated region, in 188 probands of hereditary prostate cancer (HPC) families recruited from Johns Hopkins Hospital. Two microsatellite markers surrounding PTEN were used to test the co-segregation of 10 rare variants, which may give rise to highly penetrance in HPC. Two common single nucleotide polymorphisms (SNPs) were evaluated in the $188 \mathrm{HPC}$ families using a family-based association study approach. To study low penetrant SNPs in PCa susceptibility, 33 SNPs covering PTEN were selected from the whole genome-wide association studies (GWAS) from our available case-control studies in Sweden (Cancer of the Prostate in Sweden (CAPS)) and the publicly available cancer genetic markers of susceptibility (CGEMS) study. Germ-line copy-number variations (CNVs) in PTEN were assessed in CAPS. Co-segregation of germ-line variants and PCa was not observed among HPC families and no significant differences in the allele frequencies were observed in sporadic cases and controls, aggressive and non-aggressive PCa $(P>0.05)$. These results suggest that germ-line variants in PTEN do not have an important role in PCa susceptibility.

Journal of Human Genetics (2011) 56, 496-502; doi:10.1038/jhg.2011.48; published online 2 June 2011

Keywords: co-segregation; family-based association; population-based association; prostate cancer; PTEN; sequencing

\section{INTRODUCTION}

Located on chromosome $10 \mathrm{q} 23$, PTEN is frequently deleted in multiple tumors including prostate cancer (PCa). PTEN has been shown to be involved in many cellular functions including apoptosis, cell-cycle control, cell adhesion and migration; however, the primary function of PTEN is to dephosphorylate and inactivate the second messenger PIP3. ${ }^{1}$ It exerts its tumor suppressor function by acting as a negative regulator in the PI3K signaling pathway. ${ }^{1}$ Loss of PTEN has been shown to contribute to PCa development in mouse models, ${ }^{2-5}$ and mutations or deletions of PTEN have been found to occur frequently in PCa in human, especially in advanced disease. $^{6-10}$

Deleterious germ-line variants in PTEN were not found to contribute to PCa susceptibility in previous studies, including muta- tion screening, linkage studies and genome-wide association (GWA) studies in case-control sporadic PCa populations. ${ }^{1-16}$ Some limitations of these studies were small sample size, low-coverage of the PTEN gene for mutation screening and the lack of evaluation of co-segregation of PTEN germ-line variants and PCa risk. In this report, a comprehensive assessment of germ-line variants in the PTEN gene for PCa susceptibility was carried out, which included mutation screening of a sizeable PTEN region in a large hereditary prostate cancer (HPC) sample, co-segregation analysis of high penetrant variants (rare variants with a minor allele frequency $\geqslant 5 \%$ ) in HPC families, association analysis of low penetrant variants (common single nucleotide polymorphisms (SNPs) with a minor allele frequency $<5 \%$ ) in sporadic PCa and evaluation of copy-number variations (CNVs) in PTEN in sporadic PCa populations.

\footnotetext{
${ }^{1}$ Center for Cancer Genomics, Wake Forest University School of Medicine, Winston-Salem, NC, USA; ${ }^{2}$ Department of Cancer Biology, Wake Forest University School of Medicine, Winston-Salem, NC, USA; ${ }^{3}$ Department of Biostatistical Sciences, Wake Forest University School of Medicine, Winston-Salem, NC, USA; ${ }^{4}$ Center for Genomics and Personalized Medicine Research, Wake Forest University School of Medicine, Winston-Salem, NC, USA; ${ }^{5}$ Department of Urology, Johns Hopkins Medical Institutions, Baltimore, MD, USA and ${ }^{6}$ Department of Medical Epidemiology and Biostatistics, Karolinska Institutet, Stockholm, Sweden

Correspondence: Dr J Xu, Center for Cancer Genomics, Center for Genomics and Personalized Medicine Research, Wake Forest University School of Medicine, Medical Center Boulevard, Winston-Salem, NC 27157, USA.

E-mail: jxu@wfubmc.edu
}

Received 15 December 2010; revised 7 April 2011; accepted 14 April 2011; published online 2 June 2011 


\section{MATERIALS AND METHODS}

\section{Subjects}

Hereditary prostate cancer (HPC) families. A detailed description of the HPC study families has been presented in previous work. ${ }^{17}$ In total, 188 HPC families with at least three first-degree relatives having a diagnosis of PCa were recruited into the study by investigators at the Brady Urology Institute at Johns Hopkins Hospital (JHH, Baltimore, MD, USA). The mean age at diagnosis for these individuals was 64.3 years. Approximately $91.8 \%$ of the families were Caucasian and 9.2\% were Black. The average number of affected individuals per family was 5.08 . The number of families with 3,4 and 5 or more affected individuals was 28,47 and 113, respectively. Probands were selected based on the following priorities: affected member with metastatic disease, local invasion, highest Gleason score, highest preoperative prostate-specific antigen (PSA) levels and youngest age at diagnosis.

JHH case-control population. The 1527 cases were from a consecutive series of men who underwent treatment for PCa at JHH. Diagnosis of PCa of the cases was confirmed by pathology reports. Paraffin-embedded tissue and frozen tissue were available for $95 \%$ and $35 \%$ of cases, respectively. The mean age, PSA, Gleason Score and stage for the cases were 58.5 years, $7.9 \mathrm{ng} \mathrm{ml}^{-1}, 6.8$ and 1.5 , respectively. The 482 unaffected controls were recruited from a series of consecutive men that participated in PCa screenings at the Brady Urological Institute at JHH. Mean age and PSA for the controls were 57.5 years and $1.6 \mathrm{ng} \mathrm{ml}^{-1}$. DNA from blood samples was available for all cases and controls.

Cancer of the prostate in Sweden (CAPS) population. The population-based, case-control study in Sweden, named Cancer Prostate in Sweden (CAPS) was used to complete fine-mapping in the study. ${ }^{18}$ Cases with pathologically

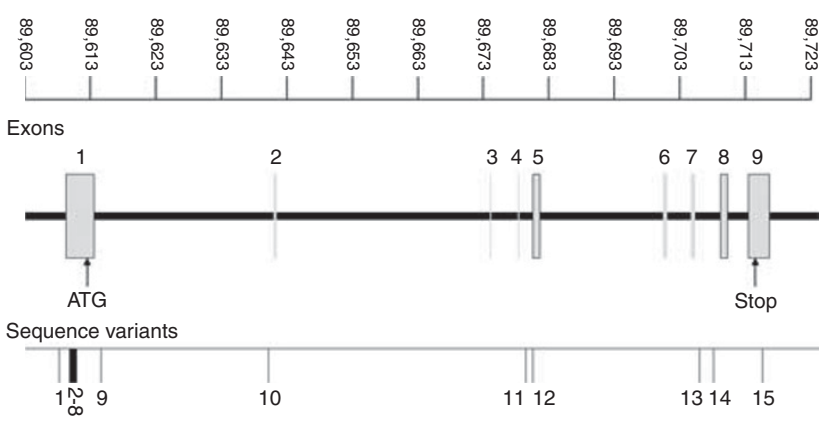

Figure 1 PTEN gene structure with sequence variants indicated (map distance in $\mathrm{kb}$ ); 15 sequence variants were found. Physical location: 89613715-89718382 bp, NCBI Build 35. diagnosed PCa were recruited from regional cancer registries in Sweden, between July 2001 and October 2003. DNA from blood samples, tumor-node metastasis stage, Gleason grade (biopsy) and PSA levels were available for 2899 cases. Controls were recruited concurrently from the Swedish Population Registry and matched with cases according to age and geographic region. DNA from blood samples was available for 1722 controls. Subsets of the CAPS population were used for two genome-wide association study (GWAS) studies: GASP1 and GASP2. GASP1 included 500 cases and 500 controls and GASP2 included 1500 cases and 500 controls.

Cancer genetic markers of susceptibility (CGEMS) population. The published data from the National Cancer Institute (CGEMS) study was also used. ${ }^{19,20}$ The study population was the Prostate, Lung, Colon and Ovarian (PLCO) Cancer Screening Trial and it included 1177 cases and 1105 controls.

\section{Sequencing and genotyping of SNP and microsatellite markers}

The entire genomic sequence of PTEN was re-sequenced, including the $\sim 2 \mathrm{~kb}$ region of the promoter, all coding regions, the $3^{\prime}$ and $5^{\prime}$ untranslated regions, and regions that were evolutionarily conserved from multiple species comparison in probands $(n=188)$ of the HPC families. Figure 1 outlines the gene organization of PTEN and Table 1 lists the primers used to amplify the PCR products, the sizes of amplified PCR fragments and the annealing temperature for each pair of primers. All reactions were performed using Taq polymerase (Invitrogen, Carlsbad, CA, USA) at a $10-\mu \mathrm{l}$ scale consisting of $20 \mathrm{ng}$ genomic DNA, $0.2 \mu \mathrm{moll}^{-1}$ of each primer, $0.2 \mathrm{mmoll}^{-1}$ of each deoxynucleotide triphosphate, $1.5 \mathrm{mmoll}^{-1} \mathrm{MgCl}_{2}, 20 \mathrm{mmoll}^{-1}$ Tris- $\mathrm{HCl}, 50 \mathrm{mmoll}^{-1} \mathrm{KCl}$ and 0.5 units Taq polymerase (Invitrogen). PCR cycling conditions were as follows: $94^{\circ} \mathrm{C}$ for $3 \mathrm{~min}$ followed by 33 cycles of $94^{\circ} \mathrm{C}$ for $30 \mathrm{~s}$, a specified annealing temperature for $30 \mathrm{~s}$, and $72{ }^{\circ} \mathrm{C}$ for $30 \mathrm{~s}$, with a final extension of $72^{\circ} \mathrm{C}$ for $6 \mathrm{~min}$. The PCR products were purified using the QuickStep PCR Purification kit (Edge BioSystems, Gaithersburg, MD, USA) to remove deoxynucleotide triphosphates and excess primers. All sequencing reactions were carried out using dye terminator chemistry (BigDye, ABI, Foster City, CA, USA) and then precipitated using $75 \pm 5 \%$ ethanol. Samples were loaded onto an ABI 3700 DNA analyzer after adding $8 \mu \mathrm{l}$ formamide. SNPs were identified using Sequencher software version 4.0.5 (Gene Codes, Ann Arbor, MI, USA).

Genotyping for the two common SNPs (rs12573787 and rs1903858) was carried out using the MassARRAY system (Sequenom, San Diego, CA, USA) in the 188 HPC families for which DNA samples were available. The SNP rs1903858 was selected to be genotyped in an additional 1527 sporadic PCa cases and 482 unaffected controls from the JHH case-control population. PCR and extension reactions were carried out according to the manufacturer's instructions and extension product sizes were determined by mass spectrometry.

The microsatellite markers D10S1686 and D10S185, which lie on either side of the PTEN gene in the region of $100.5 \sim 102.5 \mathrm{cM}$ and span an interval of

Table 1 Primers used for mutational analysis and SNPs identification in PTEN (89613 715-89718382 bp), NCBI Build 35

\begin{tabular}{|c|c|c|c|c|}
\hline$P C R I D$ & Forward primer $\left(5^{\prime}-3^{\prime}\right)$ & Reverse primer $\left(5^{\prime}-3^{\prime}\right)$ & PCR product (bp) & Annealing temperature \\
\hline 1 & AAGGGGGAATCTCTAGGCAA & AGCATGCCCAGTGTAGCTG & 453 & 62 \\
\hline 2 & AGCAAGTGCAGCTGCAGG & AGAGGCTGCACGGTTAGAAA & 579 & 62 \\
\hline 3 & CTCTCAGCGCCTGTGAGC & GGACTTGGCGGTAGCTGAT & 638 & 62 \\
\hline 4 & CCAGTCGCTGCAACCATC & TGCAACCAGGCAAGAGTTC & 473 & 68 \\
\hline 5 & CTGTCCATGTGGAAGTTACCTT & CTGTATCCCCCTGAAGTCCA & 460 & 62 \\
\hline 6 & CCATAGAAGGGGTATTTGTTTGG & CATGAATCTGTGCCAACAATG & 420 & 58 \\
\hline 7 & AAAGATTCAGGCAATGTTTGTT & ATGCTGCACTTTAGTCTTCCTG & 350 & 60 \\
\hline 8 & TTTCTCTGGAATCCAGTGTTTC & CAGATCCAGGAAGAGGAAAGG & 413 & 60 \\
\hline 9 & GCTACGACCCAGTTACCATAGC & CCAATGAGTTGAACAACAAAGC & 404 & 60 \\
\hline 10 & TTGCTTGAGATCAAGATTGCA & CTCACCAATGCCAGAGTAAGC & 446 & 60 \\
\hline 11 & TTTTGTTGACTTTTTGCAAATG & GTCAAGCAAGTTCTTCATCAGC & 442 & 62 \\
\hline 12 & AAGAGGGTCATTTAAAAGGCC & TCTGACACAATGTCCTATTGCC & 453 & 60 \\
\hline 13 & CCAGAGAATGAACCTTTTGATG & ACCCTTCGGAAACCTCTCTTAG & 520 & 68 \\
\hline 14 & GTTCTGTCACCAACTGAAGTGG & AGCATCCACAGCAGGTATTATG & 679 & 63 \\
\hline
\end{tabular}


$\sim 9.6 \mathrm{Mb}$, were genotyped in HPC families with rare sequence variants to estimate the co-segregation of sequence variants with PCa in HPC families. The genotyping method has been previously described in detail. ${ }^{21}$

\section{SNPs in the PTEN gene selected from genome-wide association study (GWAS)}

To study low penetrant SNPs in PCa susceptibility, 33 SNPs within the PTEN gene were selected from the GWAS of GASP1, GASP2 and CGEMS (see Figure 2). Data from the three existing GWAS studies, which were deposited from Hapmap (http://www.hapmap.org, rel\#21, dbSNPb125) was used to test the 33 SNPs spanning the complete PTEN gene (105.3 kb), GeneChip Human Mapping 500k Array, 5.0 Array (Affymetrix, Santa Clara, CA, USA) and HumanHap 300 BeadChips (Illumina, San Diego, CA, USA) were used for GASP1, GASP2 and CGEMS. ${ }^{19,22}$ The detection of SNPs and CNVs in the CAPS population by means of Affymetrix SNP has been previously described in detail. $^{22,23}$ Results for a subset of the 33 SNPs spanning PTEN were obtained from the GWAS (results from GASP1, GASP2 and CGEMS) and were used for the imputation of the remaining SNPs.

\section{Statistical methods}

Family-based association tests (FBAT) were performed for two SNPs (rs12573787 and rs1903858) in HPC families including 1286 affected and unaffected members, using the software package FBAT. ${ }^{24}$ Linkage and linkage disequilibrium between PCa and SNPs were assessed by FBAT based on the data from nuclear families, sibships or a combination of the two.

Parametric and non-parametric analyses were carried out to test the cosegregation of the sequence variants in the HPC families using GeneHunter Plus (http://www.broad.mit.edu/ftp/distribution/software/genehunter/). In the parametric analysis, the frequency of the susceptibility allele was assumed to be 0.003 , and penetrance for non-carriers and carriers was 0.001 and 1.0, respectively. In the recessive model, the frequency of the susceptibility allele was set to be 0.15 , and the penetrances for heterozygous carriers and homozygous carriers were assumed to be equal.

Linkage heterogeneity among families (HLOD) was estimated using HOMOG (Ott 1999). In non-parametric analysis, LOD scores were calculated using the Kong and Cox exponential allele sharing model score (KCLOD). Multipoint linkage statistics were performed at $0.5-\mathrm{cM}$ intervals across the region covering PTEN gene.

To test associations between the SNPs and CNVs and PCa, allele frequencies between cases and controls for each SNP were compared. LD structure was formed by Haploview (http://www.broadinstitute.org/mpg/haploview). The differences in allele frequencies between cases and controls were tested using standard contingency $\chi^{2}$ tests, and $P$-values were determined via $\chi^{2}$ approximation. All of the known SNPs in PTEN were imputed based on the genotyped SNP information in the HapMap Phase II data (CEU) using a computer program, IMPUTE. ${ }^{25}$ Odds ratio and $95 \%$ confidence interval were estimated using an unconditional logistic regression with adjustment for age and geographic region, assuming an additive model of inheritance.

\section{RESULTS}

\section{SNP identification}

A total of 15 genetic variants were identified in the sequenced region of PTEN in the 188 HPC probands. Five of the variants had already been previously reported (rs1044322, rs12573787, rs1903858, rs34051577 and rs1044487) and none of the 15 was located in exons (http://www.ncbi.nlm.nih.gov/SNP/snp_ref). The location and

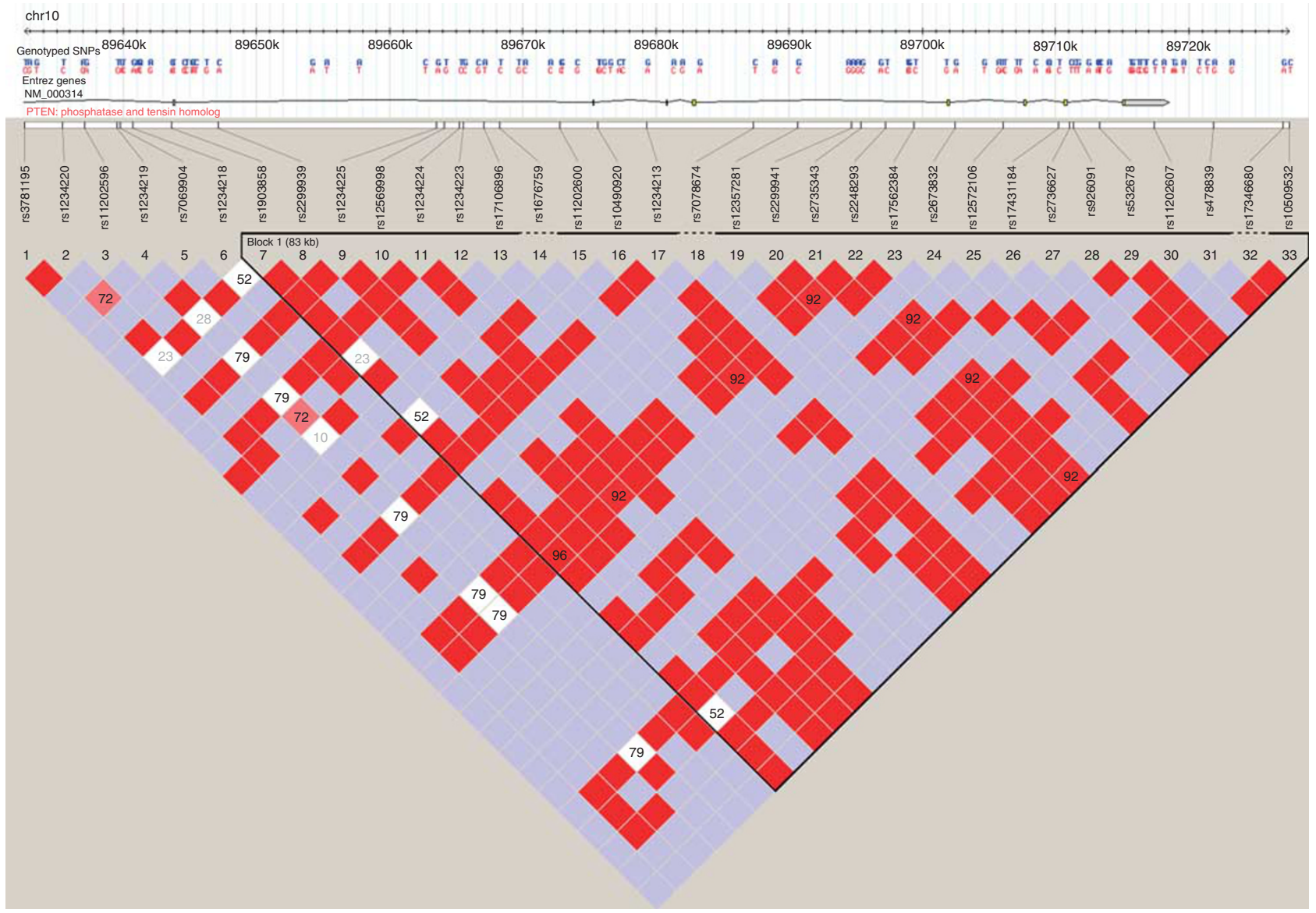

Figure 233 SNPs spanning PTEN (89623 195-89728531 bp, NCBI build 37); Color scheme is based on standard D'/LOD score option in Haploview 4.1. 
Table 2 Sequence variants of PTEN identified in 188 HPC probands

\begin{tabular}{|c|c|c|c|}
\hline Variant ID (position) $)^{\mathrm{a}}$ & Sequence variants & Position & Frequency \\
\hline $1(-1287)$ & SNP-1287C/G & Promoter & CG: $1.2 \%$; CC: $98.8 \%$; GG: 0 \\
\hline $2(-1060)$ & SNP-1060C/G & 5'UTR & CG: 0.7\%; CC:99.3\%; GG:0 \\
\hline $3(-976)$ & SNP-976G/C & 5'UTR & GC:0.7\%; GG:99.3\%; CC:0 \\
\hline $4(-904)$ & SNP-904G/A & 5'UTR & GA: 3.4\%; GG:96.6\%; AA:0 \\
\hline $5(-546)$ & INDEL-546 5GGC/6GGC & 5'UTR & $5 / 6: 2.6 \% ; 5 / 5: 97.4 \% ; 6 / 6: 0$ \\
\hline 6 rs12573787 $(-511)$ & SNP-511G/A & 5'UTR & GA:22\%; GG:77\%; AA:0.7\% \\
\hline $7(-500)$ & INDEL-500 5GGC/6GGC/7GGC & $5^{\prime} U T R$ & 5/6:0.7\%; 5/7:0.7\%; 5/5:98.6\%; 6/6:0; 7/7:0 \\
\hline $8(-461)$ & SNP-461G/A & $5^{\prime} \mathrm{UTR}$ & GA:0.7\%; GG:99.3\%; AA:0 \\
\hline $9(210)$ & SNP 210T/C & Intron 1 & TC:0.6\%; TT:99.4\%; CC:0 \\
\hline 10 rs1903858 (29460) & SNP $29460 \mathrm{C} / \mathrm{T}$ & Intron 1 & CT:47.3\%; TT:43.2\%; CC:9.5\% \\
\hline $11(66729)$ & INDEL 66729 TCTTA/_ & Intron 4 & TCTTA/-:44\%; _/_:45.6\%; TCTTA/TCTTA:10.6\% \\
\hline $12(68506)$ & INDEL 68506 9T/10T & Intron 4 & 9/10:1.8\%; 9/9:98.2\%; 10/10:0 \\
\hline $13(93573)$ & SNP 93573G/A & Intron 7 & GA:1.8\%; GG:98.2\%; AA:0 \\
\hline $14(96422)$ & INDEL 96422 15T/14T & Intron 7 & $15 / 14: 100 \% ; 15 / 15: 0 ; 14 / 14: 0$ \\
\hline $15(101068)$ & INDEL 101068 11T/10T & 3'UTR & 11/10:100\%; 11/11:0; 10/10:0 \\
\hline
\end{tabular}

Abbreviations: HPC, hereditary prostate cancer; INDEL, insertion/deletion; SNP, single nucleotide polymorphism; UTR, untranslated region.

aposition is relative to ATG translation start.

frequency of each variant are presented in Figure 1 and Table 2. Of the15 sequence variants, 5 are common variants with a minor allele frequency $\geqslant 5 \%$ and 10 are rare variants with a minor allele frequency $<5 \%$. Of the five common variants, two are SNPs and the three are insertion/deletions.

\section{Co-segregation of rare SNPs and PCa in HPC families}

LOD scores for two markers (D10S1686 and D10S185) flanking PTEN located in the region of $100.5 \sim 102.5 \mathrm{cM}$ are shown in Table 3. HLOD scores were used to present the LOD score for the family group of each SNP. Both dominant and recessive parametric analyses were carried out, and evidence of co-segregation of rare SNPs in the HPC families was not observed. Non-parametric analysis was also carried out and co-segregation of the rare SNPs and PCa was not found (data not shown).

Family-based association test (FBAT)

Two common SNPs, rs12573787 and rs1903858, which were in the $5^{\prime}$ untranslated region and intron 1, were further genotyped in the FBAT. An association between the two variants and PCa was not observed, suggesting the variants were not overtransmitted from parents to affected sons in the 188 HPC families including 1286 affected and unaffected family members (Table $4, P>0.05$ ). Subset analysis of FBAT were also carried out by breaking the families down by the number of affected males (number $=3,4$ and $\geqslant 5$ ) and age at onset $(<65$ or $>65)$. None of these sub groups showed significant association for these two SNPs in FBAT analysis (all $P>0.05)$.

\section{Association test in sporadic PCa cases and controls}

The SNP rs1903858 was selected for association analysis among sporadic PCa cases and controls. The frequency of rs1903858 is 0.31 in cases $(n=1527)$ and 0.30 in controls $(n=482)$ from the JHH population. No significant difference in the allele frequencies was found between sporadic PCa cases and controls $(P=0.592)$.

Of the 33 SNPs that spanned the PTEN gene that were selected for association testing with sporadic PCa, there were 28 common SNPs $(\geqslant 5 \%$ frequency) identified with an average spacing of one common SNP every $\sim 3.7 \mathrm{~kb}$. In the PTEN gene, four SNPs were
Table 3 Co-segregation of 10 rare SNPs in PTEN in HPC families

\begin{tabular}{|c|c|c|c|c|c|c|c|}
\hline \multirow[b]{3}{*}{ Variant ID } & \multirow[b]{3}{*}{$\begin{array}{l}\text { Family } \\
\text { number }\end{array}$} & \multicolumn{6}{|c|}{$\angle O D$ score (HLOD) } \\
\hline & & \multicolumn{3}{|c|}{ Dominant } & \multicolumn{3}{|c|}{ Recessive } \\
\hline & & $\begin{array}{c}100.5 \\
c M\end{array}$ & $\begin{array}{c}101.5 \\
c M\end{array}$ & $\begin{array}{c}102.5 \\
c M\end{array}$ & $\begin{array}{c}100.5 \\
c M\end{array}$ & $\begin{array}{c}101.5 \\
c M\end{array}$ & $\begin{array}{c}102.5 \\
c M\end{array}$ \\
\hline 1 & 2 & 0.09 & 0.11 & 0.12 & 0.08 & 0.07 & 0.07 \\
\hline 2 & 1 & 0 & 0 & 0 & 0 & 0 & 0 \\
\hline 3 & 1 & 0 & 0 & 0 & 0 & 0 & 0 \\
\hline 4 & 5 & 0 & 0 & 0 & 0 & 0 & 0 \\
\hline 5 & 4 & 0 & 0 & 0 & 0 & 0 & 0 \\
\hline 7 & 2 & 0.03 & 0.03 & 0.03 & 0.04 & 0.04 & 0.03 \\
\hline 8 & 1 & 0 & 0 & 0 & 0 & 0 & 0 \\
\hline 9 & 1 & 0 & 0 & 0 & 0 & 0 & 0 \\
\hline 12 & 3 & 0 & 0 & 0.02 & 0 & 0.01 & 0.02 \\
\hline 13 & 3 & 0 & 0 & 0 & 0 & 0 & 0 \\
\hline All_above_fam & 23 & 0 & 0 & 0 & 0 & 0 & 0 \\
\hline
\end{tabular}

Abbreviations: HPC, hereditary prostate cancer; SNP, single nucleotide polymorphism.

Table 4 Results of the FBATs for two common SNPs in PTEN

\begin{tabular}{lccccc}
\hline SNPs & $\begin{array}{c}\text { Chromosome } \\
\text { position }\end{array}$ & Alleles & $\begin{array}{c}\text { Risk } \\
\text { allele }\end{array}$ & $\begin{array}{c}\text { Allele } \\
\text { frequency }\end{array}$ & P-values $^{\text {a }}$ \\
\hline rs12573787 & 89613696 & A/G & A & 0.12 & 0.9 \\
rs1903858 & 89643666 & C/T & C & 0.7 & 0.99 \\
\hline
\end{tabular}

Abbreviations: FBATs, family-based association tests; SNP, single nucleotide polymorphism. aFBAT test: HPC cases vs controls.

genotyped in GASP1 and GASP2 and eight SNPs were genotyped in CGEMS GWAS studies. The remaining SNPs in PTEN were imputed based on the four SNPs genotyped in GASP1 and GASP2 GWAS or eight SNPs genotyped in CGEMS GWAS. None of the 33 SNPs (genotyped or imputed) was found to be significantly associated with PCa susceptibility in any these studies, shown as in Table $5(P>0.05)$. The 33 SNPs were also tested for 
their association with PCa aggressiveness in CGEMS and GASP2 populations (all cases in GASP1 population are aggressive). None of them was found to be significantly associated with aggressiveness in these two populations, as shown in Table 6 (all $P>0.05$ ).

\section{Copy-number variations (CNVs) analysis}

In addition to testing the association of germ-line SNPs and PCa susceptibility, germ-line CNVs in PTEN and the association with sporadic $\mathrm{PCa}$ was also evaluated with three SNP probes (rs12569998, rs11202600 and rs17107001) at PTEN region on GeneChip Human Mapping 5.0 Array (Affymetrix). The CNV defined by the three probes in PTEN was not associated with PCa risk from GWAS in CAPS population (data not shown).

\section{DISCUSSION}

In this report, germ-line sequence variants of PTEN in both HPC and sporadic prostate caner were comprehensively evaluated. The PTEN gene was screened, including the $5^{\prime}$ untranslated region, all nine exons, exon-intron junctions and $3^{\prime}$ untranslated regions, in HPC probands.
The co-segregation of PTEN mutations/sequence variants in HPC families and association in a sporadic PCa population were also evaluated. To summarize, 10 rare and 5 common variants were identified by sequencing, however, we were unable to identify any potentially important mutations, such as mutations in exons from germ-line DNA samples of 188 HPC probands. There was no statistically significant difference in allele frequencies of the two common SNPs, rs12573787 and rs1903858, between cases and controls after genotyping 1286 HPC members and no statistical difference in the allele frequency of one common SNP after genotyping in 1527 PCa cases and 482 controls. Additionally, there was no evidence of cosegregation of variants in PTEN in the HPC families. The 33 SNPs that spanned the PTEN gene showed no association between PTEN and PCa susceptibility in the CAPS and CGEMS population. None of the 33 SNPs was shown to be associated with aggressiveness of PCa. Finally, in the results of evaluating CNVs in PTEN from the available data in CAPS, there were no detectable CNV changes associated with $\mathrm{PCa}$ (data not shown). The results suggest that PTEN gene variants do not have an effect on PCa susceptibility and aggressiveness.

Table 5 Frequency of 33 SNPs spanning PTEN in three GWAS studies

\begin{tabular}{|c|c|c|c|c|c|c|c|c|c|c|c|c|c|c|c|c|c|}
\hline \multirow[b]{2}{*}{ SNP } & \multirow[b]{2}{*}{ Position } & \multirow{2}{*}{$\begin{array}{l}\text { Minor } \\
\text { allele }\end{array}$} & \multicolumn{5}{|c|}{ CGEMS (1177 cases/1105 controls) } & \multicolumn{5}{|c|}{ GASP1 (500 cases/500 controls) } & \multicolumn{5}{|c|}{ GASP2 (1500 cases/500 controls) } \\
\hline & & & $F_{-} A^{\boldsymbol{\Lambda}}$ & $F_{-} U^{\boldsymbol{\Delta}}$ & $p^{a}$ & $O R^{a}$ & Status & $F_{-} A^{\boldsymbol{\Lambda}}$ & $F_{-} U^{\mathbf{\Delta}}$ & $P^{a}$ & $O R^{a}$ & Status & $F_{-} A^{\boldsymbol{\Lambda}}$ & $F_{-} U^{\mathbf{\Lambda}}$ & $P^{a}$ & $O R^{a}$ & Status \\
\hline rs3781195 & 89632589 & $\mathrm{G}$ & 0.03 & 0.02 & 0.81 & 1.05 & Imputed & 0.03 & 0.01 & 0.011 & 2.29 & Imputed & 0.02 & 0.02 & 0.79 & 1.07 & Imputed \\
\hline rs1234220 & 89635453 & $\mathrm{G}$ & 0.10 & 0.10 & 0.63 & 1.05 & Original & 0.11 & 0.09 & 0.19 & 1.22 & Imputed & 0.10 & 0.12 & 0.17 & 0.85 & Imputed \\
\hline rs1 1202596 & 89637134 & $A$ & 0.09 & 0.08 & 0.70 & 1.04 & Original & 0.04 & 0.04 & 0.57 & 0.86 & Imputed & 0.05 & 0.05 & 0.95 & 0.99 & Imputed \\
\hline rs1234219 & 89639557 & $\mathrm{G}$ & 0.08 & 0.07 & 0.60 & 1.06 & Imputed & 0.06 & 0.06 & 0.96 & 1.01 & Imputed & 0.06 & 0.08 & 0.24 & 0.84 & Imputed \\
\hline rs7069904 & 89639818 & $A$ & 0.02 & 0.02 & 0.16 & 1.34 & Imputed & 0.02 & 0.01 & 0.046 & 2.72 & Imputed & 0.01 & 0.00 & 0.61 & 1.33 & Imputed \\
\hline rs1234218 & 89640739 & $\mathrm{~T}$ & 0.07 & 0.07 & 0.84 & 1.02 & Imputed & 0.05 & 0.06 & 0.74 & 0.94 & Imputed & 0.06 & 0.07 & 0.24 & 0.84 & Imputed \\
\hline rs1903858 & 89643666 & $G$ & 0.31 & 0.28 & 0.03 & 1.16 & Imputed & 0.29 & 0.30 & 0.78 & 0.97 & Imputed & 0.27 & 0.28 & 0.95 & 0.99 & Imputed \\
\hline rs2299939 & 89647130 & A & 0.19 & 0.19 & 0.63 & 1.04 & Original & 0.08 & 0.10 & 0.24 & 0.79 & Imputed & 0.09 & 0.08 & 0.45 & 1.14 & Imputed \\
\hline rs1234225 & 89663549 & $\mathrm{~T}$ & 0.35 & & 0.08 & & & & & & 0.96 & & 0.33 & & & 0.93 & \\
\hline rs12569998 & 89664137 & G & 0.14 & 0.13 & 0.29 & 1.10 & & & & & 0.98 & & & & & 1.04 & Original \\
\hline rs1234224 & 89665276 & $G$ & 0.35 & 0.32 & 0.08 & 1.12 & Imputed & 0.34 & 0.35 & 0.67 & 0.96 & Imputed & 0.33 & & 0.39 & 0.93 & \\
\hline rs1234223 & 8966 & C & 0.10 & & 0.70 & 1.04 & & & & & 1.21 & & & & & 0.85 & \\
\hline rs17106896 & 89667087 & $\mathrm{~T}$ & 0.05 & 0.04 & 0.23 & 1.19 & & 0.02 & 0.01 & 0.049 & 2.69 & & 0.01 & & 0.40 & 1.59 & \\
\hline rs1676759 & 89668276 & $\mathrm{G}$ & 0.03 & 0.02 & 0.72 & 1.07 & & 0.01 & 0.01 & 0.58 & 1.35 & Imputed & 0.01 & 0.00 & 0.34 & 1.68 & Imputed \\
\hline rs11202600 & 89672813 & C & 0.07 & 0.07 & 0.84 & 1.02 & & 0.07 & 0.07 & 0.98 & 1.00 & Original & 0.08 & 0.10 & 0.053 & 0.79 & Original \\
\hline rs10490920 & 89675623 & C & 0.14 & 0.13 & 0.29 & 1.10 & Imputed & 0.14 & 0.14 & 0.82 & 0.97 & Imputed & 0.13 & 0.13 & 0.75 & 1.04 & Imputed \\
\hline rs1234213 & 89679301 & $A$ & 0.35 & 0.32 & 0.07 & 1.12 & Imputed & 0.34 & 0.35 & 0.66 & 0.95 & Imputed & 0.33 & 0.35 & 0.39 & 0.93 & Imputed \\
\hline rs7078674 & 89687333 & $\mathrm{~T}$ & 0.00 & 0.00 & NA & NA & Imputed & 0.00 & 0.00 & NA & NA & Imputed & 0.00 & 0.00 & NA & NA & Imputed \\
\hline rs12357281 & 89690651 & C & 0.09 & 0.08 & 0.75 & 1.04 & Imputed & 0.04 & 0.04 & 0.65 & 0.89 & Imputed & 0.05 & 0.05 & 0.88 & 0.97 & Imputed \\
\hline rs2299941 & 89694699 & $\mathrm{G}$ & 0.14 & 0.13 & 0.23 & 1.11 & Imputed & 0.14 & 0.14 & 0.99 & 1.00 & Imputed & 0.13 & 0.12 & 0.87 & 1.02 & Imputed \\
\hline rs2735343 & 89695409 & C & 0.35 & 0.32 & 0.09 & 1.11 & Imputed & 0.34 & 0.35 & 0.65 & 0.95 & Imputed & 0.33 & 0.35 & 0.37 & 0.93 & Imputed \\
\hline rs2248293 & 89697245 & C & 0.35 & 0.32 & 0.07 & 1.12 & Original & 0.31 & 0.31 & 0.98 & 1.00 & Imputed & 0.30 & 0.33 & 0.16 & 0.88 & Imputed \\
\hline rs17562384 & 89699396 & C & 0.19 & 0.19 & 0.59 & 1.04 & Imputed & 0.08 & 0.10 & 0.25 & 0.79 & Imputed & 0.09 & 0.08 & 0.44 & 1.14 & Imputed \\
\hline rs2673832 & 89702453 & G & 0.11 & 0.11 & 0.92 & 0.99 & Original & 0.01 & 0.02 & 0.22 & 0.56 & Imputed & 0.02 & 0.01 & 0.38 & 1.35 & Imputed \\
\hline rs12572106 & 89706087 & C & 0.14 & 0.13 & 0.27 & 1.10 & Imputed & 0.14 & 0.14 & 0.82 & 0.97 & Imputed & 0.13 & 0.13 & 0.75 & 1.04 & Imputed \\
\hline rs17431184 & 89710231 & C & 0.19 & 0.19 & 0.60 & 1.04 & Imputed & 0.08 & 0.10 & 0.25 & 0.79 & Imputed & 0.09 & 0.08 & 0.43 & 1.15 & Imputed \\
\hline rs2736627 & 89711074 & C & 0.09 & 0.10 & 0.43 & 0.92 & Imputed & 0.01 & 0.02 & 0.22 & 0.56 & Imputed & 0.02 & 0.01 & 0.38 & 1.35 & Imputed \\
\hline rs926091 & 89711392 & $\mathrm{~T}$ & 0.14 & 0.13 & 0.27 & 1.10 & Imputed & 0.14 & 0.14 & 0.82 & 0.97 & Imputed & 0.13 & 0.13 & 0.75 & 1.04 & Imputed \\
\hline rs532678 & 89713322 & $\mathrm{~T}$ & 0.35 & 0.32 & 0.08 & 1.12 & Imputed & 0.34 & 0.35 & 0.65 & 0.95 & Imputed & 0.33 & 0.35 & 0.37 & 0.93 & Imputed \\
\hline rs1 1202607 & 89717394 & $\mathrm{~T}$ & 0.07 & 0.07 & 0.99 & 1.00 & Original & 0.05 & 0.06 & 0.74 & 0.94 & Imputed & 0.06 & 0.07 & 0.24 & 0.84 & Imputed \\
\hline rs478839 & 89721850 & G & 0.40 & 0.42 & 0.41 & 0.95 & Original & 0.40 & 0.38 & 0.39 & 1.11 & Imputed & 0.38 & 0.37 & 0.89 & 1.01 & Imputed \\
\hline rs17346680 & 89727081 & $A$ & 0.04 & 0.03 & 0.29 & 1.19 & Imputed & 0.04 & 0.02 & 0.003 & 2.24 & Original & 0.07 & 0.08 & 0.11 & 0.80 & Original \\
\hline rs10509532 & 89727534 & $\mathrm{~T}$ & 0.14 & 0.13 & 0.26 & 1.10 & Original & 0.14 & 0.14 & 0.78 & 0.96 & Original & 0.13 & 0.13 & 0.75 & 1.04 & Original \\
\hline
\end{tabular}

Abbreviations: CGEMS, cancer genetic markers of susceptibility; GWAS, whole genome-wide association study; OR, odds ratio; SNPs, single nucleotide polymorphisms.

aP and $\mathrm{OR}$ are calculated based on an unconditional logistic regression with adjustment for age and geographic region, assuming an additive model of inheritance. $\mathbf{F}_{-} \mathrm{A}_{\text {, frequency in affected }}$ cases; F_U, frequency in controls. 
Table 6 Frequency of 33 SNPs spanning PTEN in 2 GWAS studies

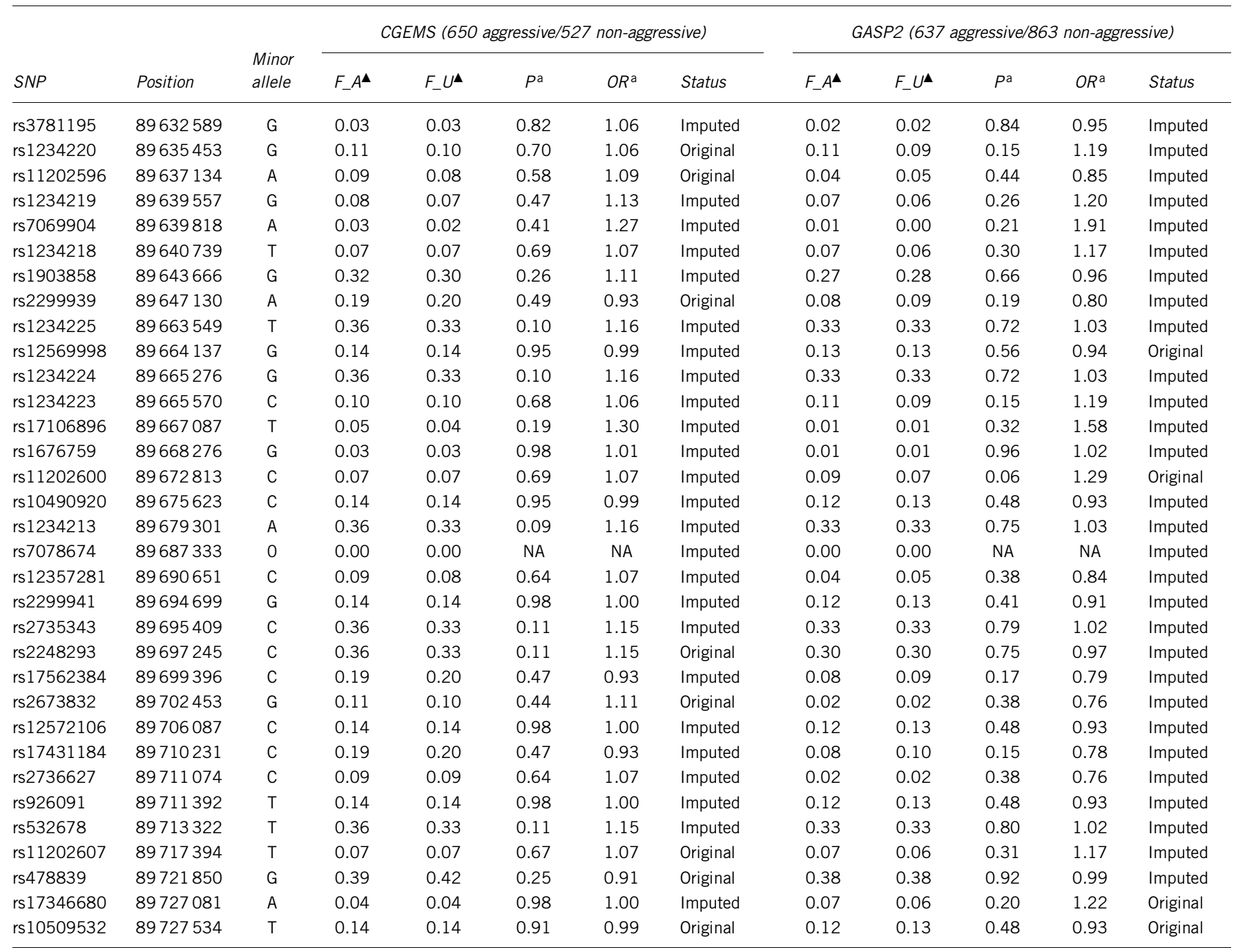

Abbreviations: CGEMS, cancer genetic markers of susceptibility; GWAS, whole genome-wide association study; OR, odds ratio; SNPs, single nucleotide polymorphisms,

aP and $\mathrm{OR}$ are calculated based on an unconditional logistic regression with adjustment for age and geographic region, assuming an additive model of inheritance. $\mathbf{A}_{\mathrm{F}} \mathrm{A}$, frequency in aggressive cases; F_U, frequency in non-aggressive cases.

This is the first time that germ-line variants of PTEN, including both rare and common variants and CNVs, were comprehensively evaluated in hereditary and sporadic PCa populations. The PTEN region was re-sequenced in a large sample size $(n=188)$ of HPC probands. No deleterious variants were found to contribute to $\mathrm{PCa}$ susceptibility, which was consistent with previous studies. ${ }^{13,14}$ Co-segregation of 10 rare variants, which were believed to give rise to high-penetrant HPC, was further evaluated by testing two microsatellite markers flanking PTEN in the HPC families. However, cosegregation was not observed in the HPC families. Common variants, which were believed to contribute low-penetrant PCa, were explored in sporadic PCa populations and no association was found between the SNPs and PCa risk, consistent with previous studies. ${ }^{15,16}$ It was also the first time that germ-line CNVs in PTEN were explored for PCa susceptibility. In our study, there were no CNV probes on PTEN region, and three SNP probes were used to define CNVs for PTEN. No CNV defined by the three SNPs at PTEN was found to be associated with sporadic PCa. Based on the available CNV information from
Hapmap, ${ }^{26}$ the nearest CNVs on the $5^{\prime}$ and $3^{\prime}$ flank region of PTEN (89613715-89718382) are 87791026-87798355 ( $\sim 1.8 \mathrm{Mbp}$ on $5^{\prime}$ region) and $90932947-90938070\left(\sim 1.2 \mathrm{Mbp}\right.$ on $3^{\prime}$ region), respectively. It is possible that CNVs at PTEN can be detected by microarrays with higher resolution.

To date, there have not been any germ-line variants in PTEN found to contribute to PCa susceptibility. However, the possibility can not be eliminated that germ-line and/or somatic sequence variants in PTEN may be associated with PCa aggressiveness. In the literature the presence of TMPRSS2: ERG fusion and a PTEN deletion was reported to be associated with a more aggressive form of $\mathrm{PCa} .{ }^{27} \mathrm{In}$ our studies, however, germ-line sequence variants of PTEN did not serve as indicator for the aggressiveness of PCa from the GWAS studies in both CGEM and GASP2 population. Therefore, germ-line sequence variants of PTEN could not serve as an indicator for the aggressiveness of PCa.

In conclusion, this study suggests that PTEN germ-line SNPs are unlikely to have an important role in hereditary and non-hereditary PCa susceptibility. 
1 Di Cristofano, A. \& Pandolfi, P. P. The multiple roles of PTEN in tumor suppression. Cell 100, 387-390 (2000)

2 Di Cristofano, A., Pesce, B., Cordon-Cardo, C. \& Pandolfi, P. P. PTEN is essential for embryonic development and tumor suppression. Nature Genet. 19, 348-355 (1998).

3 Wang, S., Gao, J., Lei, Q., Rozengurt, N., Pritchard, C., Jiao, J. et al. Prostate-specific deletion of the murine PTEN tumor suppressor gene leads to metastatic prostate cancer. Cancer Cell. 4, 209-221 (2003).

4 Abate-Shen, C., Banach-Petrosky, W. A., Sun, X., Economides, K. D., Desai, N., Gregg, J. P. et al. Nkx3.1; Pten mutant mice develop invasive prostate adenocarcinoma and lymph node metastases. Cancer Res. 63, 3886-3890 (2003).

5 Halvorsen, O., Haukaas, S. A. \& Akslen, L. A. Combined loss of PTEN and p27 expression is associated with tumor cell proliferation by ki-67 and increased risk of recurrent disease in localized prostate cancer. Clin. Cancer Res. 9, 1474-1479 (2003).

6 Dong, J. T., Li, C. L., Sipe, T. W. \& Frierson, H. F Jr. Mutations of PTEN/MMAC1 in primary prostate cancers from Chinese patients. Clin. Cancer Res. 7, 304-308 (2001).

7 Simpson, L. \& Parsons, R. PTEN: life as a tumor suppressor. Exp. Cell Res. 264, 29-41 (2001).

8 Schmitz, M., Grignard, G., Margue, C., Dippel, W., Capesius, C., Mossong, J. et al. Complete loss of PTEN expression as a possible early prognostic marker for prostate cancer metastasis. Int. J. Cancer 120, 1284-1292 (2006).

9 Garraway, L. A., Widlund, H. R., Rubin, M. A., Getz, G., Berger, A. J., Ramaswamy, S. et al. Integrative genomic analyses identify MITF as a lineage survival oncogene amplified in malignant melanoma. Nature 436, 117-122 (2005).

10 Verhagen, P. C., Duijin, P. W., Hermans, K. G., Looijenga, L. H., Van Gurp, R. J., Stoop, H. et al. The PTEN gene in locally progressive prostate cancer is preferentially inactivated by bi-allelic gene deletion. J. Pathol. 208, 699-707 (2006).

11 Witte, J. S., Goddard, K. A., Conti, D. V., Elston, R. C., Lin, J., Suarez, B. K. et al. Genomewide scan for prostate cancer-aggressiveness loci. Am. J. Hum. Genet. 67, 92-99 (2000).

12 Gibbs, M., Stanford, J. L., Jarvik, G. P., Janer, M., Badzioch, M., Peters, M. A. et al. A genomic scan of families with prostate cancer identifies multiple regions of interest. Am. J. Hum. Genet. 67, 100-109 (2000).

13 Cooney, K. A., Tsou, H. C., Petty, E. M., Miesfeldt, S., Ping, X. L., Gruener, A. C. et al. Absence of PTEN germ-line mutations in men with a potential inherited predisposition to prostate cancer. Clin. Cancer Res. 5, 1387-1391 (1999).

14 Forrest, M. S., Edwards, S. M., Hamoudi, R. A., Dearnaley, D. P., Arden-Jones, A., Dowe, A. et al. No evidence of germline PTEN mutations in familial prostate cancer. J. Med. Genet. 3, 210-212 (2000).
15 Bar-Shira, A., Matarasso, N., Rosner, S., Bercovich, D., Matzkin, H. \& Orr-Urtreger, A. Mutation screening and association study of the Candidate prostate cancer susceptibility genes MSR1, PTEN, and KLF6. Prostate 66, 1052-1060 (2006).

16 Haiman, C. A., Stram, D. O., Cheng, I., Giorgi, E. E., Pooler, L., Penney, K. et al. Common genetic variation at PTEN and risk of sporadic breast and prostate cancer. Cancer Epidemiol. Biomarkers. Prev. 15, 1021-1025 (2006).

17 Xu, J., Gillanders, E. M., Isaacs, S. D., Chang, B. L., Wiley, K. E., Zheng, S. L. et al. Genome-wide scan for prostate cance rsusceptibility genes in the Johns Hopkins hereditary prostate cancer families. Prostate 57, 320-325 (2003).

18 Zheng, S. L., Sun, J., Wiklund, F., Smith, S., Stattin, P., Li, G. et al. Cumulative association of five genetic variants with prostate cancer. N. Engl. J. Med. 358, 910-919 (2008).

19 Yeager, M., Orr, N., Hayes, R. B., Jacobs, K. B., Kraft, P., Wacholder, S. et al. Genomewide association study of prostate cancer identifies a second risk locus at $8 q 24$. Nat. Genet. 39, 645-649 (2007).

20 Thomas, G., Jacobs, K. B., Yeager, M., Kraft, P., Wacholder, S., Orr, N. et al. Multiple loci identified in a genome-wide association study of prostate cancer. Nat. Genet. 40, 310-315 (2008)

21 Amundadottir, L. T., Sulem, P., Gudmundsson, J., Helgason, A., Baker, A., Agnarsson, B. A. et al. A common variant associated with prostate cancer in European and African populations. Nat. Genet. 38, 652-658 (2006).

22 Duggan, D., Zheng, S L., Knowlton, M., Benitez, D., Dimitrov, L., Wiklund, F. et al. Two genome wide association studies of aggressive prostate cancer implicate putative prostate tumor suppressor gene DAB2IP. J. Nat/ Cancer Inst. 99, 1836-1844 (2007).

23 Liu, W., Sun, J., Li, G., Zhu, Y., Zhang, S., Kim, S. T. et al. Association of a germ-line copy number variation at 2 p24.3 and risk for aggressive prostate cancer. Cancer Res. 69, 2176-2179 (2009).

24 Laird, N. M., Horvath, S. \& Xu, X. Implementing a unified approach to family-based tests of association. Genet. Epidemiol. 19, S26-42 (2000).

25 Marchini, J., Howie, B., Myers, S., McVean, G. \& Donnelly, P. A new multipoint method for genome-wide association studies by imputation of genotypes. Nat. Genet. 39, 906-913 (2007).

26 McCarroll, S. A., Kuruvilla, F. G., Korn, J. M., Cawley, S., Nemesh, J., Wysoker, A. et al. Integrated detection and population-genetic analysis of SNPs and copy number variation. Nat. Genet. 40, 1166-1174 (2008).

27 Yoshimoto, M., Joshua, A. M., Cunha, I. W., Coudry, R. A., Fonseca, F. P., Ludkovski, O. et al. Absence of TMPRSS2:ERG fusions and PTEN losses in prostate cnacer is associated with a favorable outcome. Mod. Pathol. 23, 1-10 (2008). 